\title{
Matrix Metalloproteinases-3 Baseline Serum Levels in Early Rheumatoid Arthritis Patients without Initial Radiographic Changes: A Two-Year Ultrasonographic Study
}

\author{
Slavica Z. Prodanovic ${ }^{a, b}$ Goran Radunovic ${ }^{a, b}$ Dragan Babic ${ }^{b, c}$ Biljana Ristic ${ }^{c}$ \\ Mirjana Sefik-Bukilica ${ }^{a}$ b Maja Zlatanovic ${ }^{a}$ Katarina Simic-Pasalic ${ }^{a}$ b \\ Srdjan Seric ${ }^{a}$ Nada Vujasinovic-Stupar ${ }^{a, b}$ Janko Samardzic ${ }^{b, d}$ \\ Nemanja Damjanov ${ }^{a, b}$

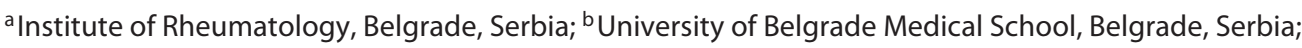 \\ 'Institute of Medical Statistics and Informatics, Belgrade, Serbia; ${ }^{\mathrm{d}}$ Institute of Pharmacology, \\ Clinical Pharmacology and Toxicology, Belgrade, Serbia
}

\section{Keywords}

Early rheumatoid arthritis · Predictive biomarkers · Matrix metalloproteinases-3 $\cdot$ Bone erosions $\cdot$ Hand joints

\footnotetext{
Abstract

Objective: To investigate the association of high baseline serum levels of metalloproteinases-3 (MMP-3) with structural damage to hand and feet joints, assessed by ultrasonography (US), in patients with early, treatment-naïve rheumatoid arthritis (RA), without initial X-ray-visible erosions, during 24 months follow-up. Methods: Sixty-three early RA (European League Against Rheumatism/American College of Rheumatology 2010), disease-modifying anti-rheumatic drugs/glucocorticoid naïve patients (mean age $53.4 \pm 14.1$ ) with symptom duration $\leq 12$ months, had baseline serum levels of MMP-3 tested. OMERACT US group definition was used to
}

\begin{tabular}{ll}
\hline KARGER & $\begin{array}{l}\text { (C) 2018 The Author(s) } \\
\text { Published by S. Karger AG, Basel Open }\end{array}$ \\
E-Mail karger@karger.com & $\begin{array}{l}\text { This is an Open Access article licensed under the Creative Commons } \\
\text { www.karger.com/mpp }\end{array}$ \\
$\begin{array}{l}\text { Attribution-NonCommercial-4.0 International License (CC BY-NC) } \\
\text { (http://www.karger.com/Services/OpenAccessLicense), applicable to } \\
\text { the online version of the article only. Usage and distribution for } \\
\text { commercial purposes requires written permission. }\end{array}$
\end{tabular}

detect the presence, as well as longitudinal diameter of erosions by US at study entry and after 24 months, at the level of wrists, metacarpophalangeal (MCP2/MCP5) joints of both hands, and fifth metatarsophalangeal joints. Results: Complete data were collected from 52 out of 63 patients. High baseline serum levels of MMP-3 (MMP-3-positive) were found in 46/63 patients. 122 bone erosions in total ( 1.9 bone erosions/patients) were detected by US at baseline visit and 213 erosions (4.3/patients) after 24 months. MMP-3 positive patients had significantly higher total number of erosions than MMP-3-negative $(p=0.039)$ and higher increase in size of bone erosions in the feet but not in the hand joints after follow-up (OR 4.82 [1.23-18.9], $p=0.024$; OR 1.17 [0.3204.26], $p=0.816$ respectively). Conclusion: After 2 years of follow-up, US assessment showed a higher number of new bone erosions in MMP-3-positive compared to MMP-3-negative patients with early $\mathrm{RA}$ and no visible initial radiograph- 
ic changes. High baseline levels of MMP-3 predict significantly higher structural damage progression at the level of feet, but not at the level of hand joints.

(c) 2018 The Author(s)

Published by S. Karger AG, Basel

\section{Introduction}

Rheumatoid arthritis (RA) is a chronic disease with a high risk of functional disability [1]. Structural damage in RA is still difficult to predict and usually develops within the first 2 years of disease [2]. Known prognostic biomarkers of the radiographic progression in RA (erythrocyte sedimentation rate $[\mathrm{ESR}]$ and C-reactive protein [CRP]) are not always accurate as in some cases structural damage can progress when these biomarkers are within normal levels [3-5]. This suggests the need for additional, more sensitive predictive biomarkers of structural damage.

Data have shown that a model consisting of matrix metalloproteinases-3 (MMP-3), (MMP-3 stromelysin) performed better than ESR and CRP in predicting 8.2year radiographic progression [6]. MMP-3 is a proteolytic enzyme, which is locally produced in the inflamed joint, and released into the blood stream. Several studies have indicated that serum levels of MMP-3 correlate with MMP-3 produced by the synovium, thus reflecting the level of activity of RA [7, 8]. Therefore, the serum level of MMP-3 is considered a biomarker that reflects local inflammation of joints. Normally, there is a tight equilibrium between MMPs and their specific tissue inhibitors (TIMP) [9]. However, in pathological conditions, such as RA, an MMP/TIMP imbalance causes excessive activation of MMPs leading to massive cartilage degradation as well as consequent bone erosion formation.

Typical radiographic features of RA, such as bone erosions, may not be present in the early stages of the disease [10]. Ultrasonography (US) is an imaging modality that is highly accurate for detection and semiquantitative assessment of bone erosion in RA [11]. Even the smallest erosions, only detected in one plane by US, could be generally confirmed by CT [12]. Furthermore, US is more sensitive than X-ray in detecting erosions in RA especially in the first 2 years of disease with sensitivity and specificity comparable to magnetic resonance imaging [13, 14]. The aim of this study was to investigate the association of high baseline serum levels of MMP-3 with structural damage to hand and feet joints, assessed by US, in patients with early, treatment-naïve RA, without X-rayvisible erosions, during a 24 month follow-up.

Serum MMP-3 Levels in RA Patients without Initial Radiographic Changes

\section{Patients and Methods}

Sixty-three patients, including 9 males and 54 females, with ages from 21 to 81 years (mean $53.4 \pm 14.1$ years) with symptom duration of $\leq 12$ months (average duration $3.8 \pm 2.7$ months) and diagnosis of early RA according to the 2010 American College of Rheumatology/European League Against Rheumatism classification criteria for RA [15], were included in this prospective clinical study during 2012-2014. All patients provided written informed consent before clinical, laboratory, and US assessment at the baseline visit. The study was approved by the Medical Ethics Committee of the Institute of Rheumatology. In order to check the sensitivity and specificity of ultrasound diagnosis of bone erosions in RA, 30 healthy controls (26 females and 4 males, mean age $51 \pm 11.4$ ) were recruited among the staff of the hospital. The patients had to meet the following inclusion criteria before entering the study: (1) the absence of bone erosions (structural damage) of hands and feet on plain radiography, and (2) the patients had to be treatment-naive for diseasemodifying anti-rheumatic drugs (DMARDs) and glucocorticoids. Patients with other systemic autoimmune disorders, other joint inflammatory diseases or severe degenerative disorders were excluded.

Composite disease activity assessment (DAS-28 score) was calculated at study entry and after 24 months of follow-up. The patients filled in the Health Assessment Questionnaire (HAQ) and the HAQ disability index was calculated for each patient.

The levels of MMP-3, ESR, CRP, RF (normal level $<16 \mathrm{U} / \mathrm{mL}$ ) and anti-citrullinated protein antibodies (ACPA; normal level $<20 \mathrm{IU} / \mathrm{mL}$ ) were measured at the Institute of Rheumatology in Belgrade. Serum levels of soluble MMP-3 were measured with a human MMP-3 detection kit (AESKU Diagnostics, Wendelsheim, Germany) according to the manufacturer's instructions. This kit detects total MMP-3 (pro- and active MMP-3) in human serum. Serum samples were collected from RA patients after overnight fasting and stored at $-80^{\circ} \mathrm{C}$ until analysis. Measurements were done in duplicate. The assays were performed blindly, without knowledge of the patient's clinical status. Serum MMP-3 measurement was performed at the same time for all samples using the recommended normal cutoff range for females of 18-60 ng/ $\mathrm{mL}$ and for males of 24-120 ng/mL. A MMP-3 level above normal (for females $>60 \mathrm{ng} / \mathrm{mL}$ and for males $>120 \mathrm{ng} / \mathrm{mL}$ ) was rated as positive.

US assessment of hands and feet at the baseline visit and after 24 months was performed by the same experienced ultrasonographer (SP), who was blinded to both the clinical and laboratory findings. The assessment was performed on the same day when the diagnosis of early RA was established and repeated after 2 years of treatment, using an $18 \mathrm{MHz}$ linear probe of Esaote My Lab 70 high resolution machine. Bone erosions were detected according to the US outcome measures in RA clinical trials definition [16], and assessed at the wrist (lunate bone and styloid process at the dorsal, radial and ulnar aspect) and metacarpophalangeal joints (MCP2 and MCP5 at dorsal and radial side) of both hands. In addition, the ulnar sides of the MCP5 joints were examined. In the feet, erosions were detected at the level of the fifth (MTP5) joint on the dorsal and lateral side. US findings of bone erosions were rated as present or absent at each selected joint sites (semi-qualitative method). In order to assess the progression of preexisting erosions detected at baseline visit, the change in ex-

Med Princ Pract 2018;27:378-386 
Table 1. Laboratory, clinical, US, and radiographic findings in group of early RA patients at baseline and after 24 months of follow-up

\begin{tabular}{lcc}
\hline Parameters & At baseline & After 24 months \\
\hline ESR & $47.2 \pm 33.7(5-140)$ & $20.5 \pm 19.4(2-100)$ \\
CRP & $29.6 \pm 34.7(0-152)$ & $11.1 \pm 16.9(20-81)$ \\
RF $(K)$ & $88.8 \pm 108.2(0-640)$ & $71.3 \pm 63.7(0-204.0)$ \\
RF $(+), n(\%)$ & $42(66.7)$ & Not done \\
ACPA $(K)$ & $467.0 \pm 454.4(0-1,334.3)$ & $392.6 \pm 430.0(0.1-1,444.6)$ \\
ACPA $(+), n(\%)$ & $46(73)$ & Not done \\
MMP-3 (K) & $185.1 \pm 241.0(0-1,322.5)$ & Not done \\
DAS-28 & $5.7 \pm 1.3(3.2-8.7)$ & $3.4 \pm 1.3(1.6-7.7)$ \\
HAQ & $1.2 \pm 1.3(0-9.2)$ & $0.5 \pm 0.7(0-2.8)$ \\
Number of US erosions/patients & $1.9 \pm 1.3(0-6)$ & $4.3 \pm 1.9(1-10)$ \\
Size of US eros/patients & $1.5 \pm 0.7(1-3)$ & $1.8 \pm 0.8(1-4)$ \\
TSS & $1.6 \pm 2.2(0-6)$ & $4.7 \pm 9.9(0-50)$ \\
\hline
\end{tabular}

Values are the mean \pm SD (range).

US, ultrasonography; RA, rheumatoid arthritis; ESR, erythrocyte sedimentation rate $(\mathrm{mm} / \mathrm{h})$; CRP, C-reactive protein $(\mathrm{g} / \mathrm{L})$; RF $(\mathrm{K})$, rheumatoid factor concentration; RF (+), rheumatoid factor positivity-number of patients; ACPA $(\mathrm{K})$, anti-citrulinated peptide antibody concentration; ACPA (+), anti-citrulinated peptide antibody positivity-number of patients; MMP-3 (K), matrix metalloproteinases-3 concentration (ng/mL); DAS-28, Disease Activity Index; HAQ, Health Assessment Questionnaire; number of US erosion/patients, number of bone erosion per patients depicted by US in whole group of RA patients; size of US eros, mean size of US bone erosions in whole group of RA patients; TSS, modified total sharp score.

tract of bone erosion was recorded. The bone erosions were rated from 0 to 4 depending on the longitudinal diameter $(\mathrm{mm})$ of erosions ( 0 = no bone erosion; $1=\leq 1 \mathrm{~mm} ; 2=1 \leq 2 \mathrm{~mm} ; 3=2 \leq 3 \mathrm{~mm}$; $4>3 \mathrm{~mm})$. The increase in the number or size of bone erosions after 24 months follow-up were considered progression of structural damage in patients with early RA.

Radiographic assessment was carried out by the radiologist blinded for clinical and US findings, the same day when the clinical, US and laboratory assessment of patients were performed. A postero-anterior X-ray of the patients' hands and antero-posterior $\mathrm{X}$-ray of the patients' feet were obtained at baseline and after 2 years of follow-up. Structural damage was assessed according to the modified Van Der Heijde Sharp score [17]. The erosion scores and the joint space narrowing scores were assessed, and the modified Total Sharp Score (TSS) was calculated.

Data evaluation and statistical analysis were performed using SPSS version 16.0 software (SPSS Inc., Chicago, IL, USA). Normally distributed continuous data were summarized by mean and SD. Categorical data were analyzed using chi-square tests. Comparisons within-groups were made using the Mann-Whitney U test, McNemar, chi-square, and Willcoxon's test. Any value of $p<0.05$ was considered statistically significant. To check potential confounding factors, Mantel-Haenszel test was performed for attributive parameters (age of patients, sex, and cigarette smoking) and partial coefficient of correlation was used for testing the numeric parameters (RF concentration, ACPA concentration, and duration of RA). Binary logistic regression models were applied in order to determine predictors of affected area and number of affected joints, as dependent variables, with sociodemographic characteristics and MMP-3 level as independent variables.

\section{Results}

Complete baseline and follow-up data were collected from 52 out of $63(82.5 \%)$ patients included in the study (11 patients were lost in follow-up, among them 7 were MMP-3-positive and 4 were MMP-3-negative). The clinical, laboratory, and radiographic findings in this group of early RA patients at baseline and after 24 months of follow-up are presented in Table 1. Forty six out of 63 patients $(73 \%)$ had MMP-3 values higher than normal (positive) and 17 (27\%) were within the normal range (negative). At the study entry, there was no statistically significant difference between group of MMP-3 positive and MMP-3-negative patients regarding age, sex, disease duration, and smoking $(p=0.328, p=0.202, p=0.801$, and $p=0.123$ respectively; $p>0.05)$. The group of MMP-3 positive patients had significantly higher HAQ index than MMP-3 negative ( $p=0.002 ; p<0.05)$. The demographic characteristics of MMP-3 positive and MMP-3 negative patients are presented in Table 2. After the US examination was completed at the baseline visit, patients were treated consecutively with methotrexate $15 \mathrm{mg}$ /weekly (46 patients), sulfasalazine (5 patients), anti-malarial drugs (2 patients), prednisone $10 \mathrm{mg} /$ day (1 patient). A combination of DMARDs and glucocorticoid therapy was used in 9 of patients. During the follow-up period, 10 (8 
Table 2. Demographic characteristics of MMP-3-positive and MMP-3-negative patients

\begin{tabular}{lccc}
\hline & $\begin{array}{l}\text { MMP-3-positive patients } \\
(n=46)\end{array}$ & $\begin{array}{l}\text { MMP-3-negative patients } \\
(n=17)\end{array}$ & $p$ value \\
\hline Age, years & $54.8 \pm 13.5(23-8)$ & $50.3 \pm 15.5(21-69)$ & 0.328 \\
Females, $n(\%)$ & $41(89.1)$ & $13(76.24)$ & 0.202 \\
Males, $n(\%)$ & $5(10.8)$ & $4(23.5)$ & 0.801 \\
Symptoms duration, months & $3.2 \pm 1.7$ & $0.4 \pm 4.0$ & $0.002^{*}$ \\
HAQ & $1.5 \pm 1.4$ & $4(23.5)$ & 0.123 \\
Smoking, $n(\%)$ & $15(32.6)$ & $11(64.7)$ & \\
$\quad$ AS & $17(36.9)$ & $2(11.8)$ & \\
NS & $14(30.4)$ & & \\
\hline
\end{tabular}

MMP-3, metalloproteinases-3; HAQ, Health Assessment Questionnaire; smoking AS, active smokers; NS, nonsmokers; ES, ex smokers, Mann-Whitney/*, Wilcoxon test and chi-square test were used. ${ }^{*} p<0.05$ (Mann-Whitney test).

Table 3. Treatment of MMP-3 positive and MMP-3 negative patients after baseline visit and at 24 months of follow-up period (number of patients)

\begin{tabular}{|c|c|c|c|c|c|c|c|c|}
\hline MMP-3-positive patients & 33 & 3 & 1 & 1 & 8 & 0 & 0 & 46 \\
\hline MMP-3-positive patients & 24 & 1 & 0 & 0 & 5 & 8 & 1 & 39 \\
\hline MMP-3-negative patients & 9 & 1 & 0 & 0 & 0 & 2 & 1 & 13 \\
\hline
\end{tabular}

MMP-3, metalloproteinases-3; MTX, therapy of methotrexate; SSL, therapy of sulfsalazine, antimal, therapy of antimalarial drugs; Gl, glucocorticoid therapy; DMARDs + Gl, combination of DMARDs and glucocorticoid therapy; DMARDs + B, combination of DMARDs and biologic therapy; NT, not treated.

MMP-3-positive, 2 MMP-3-negative) patients were additionally treated with biologic DMARDs due to RA activity. At the end of the study, 33 out of 52 patients (24 of MMP3- positive, 9 of MMP-3-negative) remained on methotrexate and two on sulfasalazine (1 of MMP-3-positive, 1 of MMP-3-negative). Five patients continued to take a combination of one of the DMARDs and glucocorticoid therapy (all were MMP-3-positive). After 6 months of treatment, 2 patients (1 MMP-3-positive, 1 MMP-3-negative) decided to stop treatment with MTX (Table 3).

A total of 504 joints were assessed at each visit. Bone erosions were present in 55 (87.3\%) patients, most often in MTP5 joints, both at study entry and after 24 months of follow-up (39.6\% of patients and $61.5 \%$ respectively). One hundred twenty two bone erosions in total (1.9 bone erosions per patient) were detected at baseline visit and 213 bone erosions ( 4.3 per patient) after 24 months. At study entry, the mean size of bone erosion in whole group of our early RA patients was 1.5 (range 1-3) and 1.8 (range

Serum MMP-3 Levels in RA Patients without Initial Radiographic Changes
1-4) after 24 months (Table 1). MMP-3-positive patients had a higher (but not statistically significant) total number of bone erosions at the baseline visit in comparison with the MMP-3 negative patients $(p=0.07)$. However, after 24 months of follow-up a significantly higher total number of erosions was detected in the MMP-3-positive compared to the MMP-3-negative patients ( $p=0.039 ; p<$ 0.05 ; Table 4 ). The potential confounding factors of age, sex, smoking, RF and ACPA concentration, treatment approach, and duration of RA did not contribute to these results when Mantel-Haenszel test and the partial coefficient of correlation was performed. After 24 months of follow-up, a significant increase was observed in the number of MMP-3-positive patients with bone erosions at lunate bone and MTP5 joints $(p=0.0001 ; p<0.01)$ and a significantly increased number of MMP-3-positive patients with erosions at the level of styloid process, MCP2, and MCP5 joints $(p=0.049, p=0.001$, and $p=0.039$ respectively; $p<0.05)$ were found. Among MMP-3-nega- 
Table 4. Ultrasonographic and RA activity assessment in MMP-3-positive and MMP-3-negative patients at the baseline and after 24 months

\begin{tabular}{|c|c|c|c|}
\hline & $\begin{array}{l}\text { MMP-3-positive } \\
\text { patients, mean } \pm \mathrm{SD}\end{array}$ & $\begin{array}{l}\text { MMP-3-negative } \\
\text { patients, mean } \pm \text { SD }\end{array}$ & $p$ value \\
\hline \multicolumn{4}{|c|}{ Number of bone erosion/patients } \\
\hline At baseline & $2.6 \pm 1.4$ & $1.5 \pm 1.0$ & 0.07 \\
\hline After 24 months & $3.8 \pm 2.3$ & $2.4 \pm 2.5$ & $0.039 *$ \\
\hline \multicolumn{4}{|c|}{ Size of bone erosion/patients } \\
\hline At baseline & $1.7 \pm 0.7$ & $1.5 \pm 0.8$ & 0.592 \\
\hline After 24 months & $1.9 \pm 0.7$ & $1.6 \pm 0.4$ & 0.755 \\
\hline \multicolumn{4}{|l|}{ ESR } \\
\hline At baseline & $53.6 \pm 33.1$ & $25.9 \pm 17.4$ & $0.002^{*}$ \\
\hline After 24 months & $19.2 \pm 19.7$ & $4.0 \pm 20.1$ & $0.009 *$ \\
\hline \multicolumn{4}{|l|}{ CRP } \\
\hline At baseline & $36.6 \pm 37.4$ & $9.5 \pm 10.7$ & $0.005^{*}$ \\
\hline After 24 months & $10.7 \pm 15.4$ & $13.2 \pm 23.1$ & $0.021^{*}$ \\
\hline \multicolumn{4}{|l|}{ DAS-28 } \\
\hline At baseline & $6.0 \pm 1.3$ & $4.8 \pm 1.2$ & $0.002^{*}$ \\
\hline After 24 months & $3.5 \pm 1.1$ & $3.2 \pm 1.9$ & $0.028^{*}$ \\
\hline
\end{tabular}

tive patients, there was no significant increase in the number of patients with bone erosions in joints (styloid process, lunate bone, MCP2, MCP5 and MTP5: $p=0.125$, $p=0.125, p=0.063, p=0.500$, and $p=1.000$ respectively; $p>0.05)$. The number of MMP-3-positive and MMP3 -negative patients with erosions in joints at baseline visit and after 24 months are presented in Figures 1a and 2a.

The majority of early RA patients (both MMP-3-positive and MMP-3-negative) had small bone erosions (size $1, \leq 1 \mathrm{~mm}$ ) detected by US at baseline as well as at followup visit (Fig. 1b, 2b). The extent of bone erosion was higher in the MMP-3-positive than in MMP-3-negative patients, both at the baseline visit and after 24 months of treatment. However, it did not reach statistical significance ( $p=0.592, p=0.755$ respectively; $p>0.05$; Table 4 ). Binar logistic regression analysis showed that patients with baseline serum levels MMP-3 higher than normal had 5 times a greater chance for the increase of extent of bone erosion at the level of feet joints (OR 4.82 [1.23$18.9] ; p=0.024$ ) after 24 months. There was no significant prediction value of MMP-3-positivity for the increase in size of bone erosion at the level of hand joints (OR 1.88 [0.49-0.20], $p=0.355$ for wrist; OR 1.29 [0.35-4.73], $p=$ 0.697 for MCP2 joints; OR 2.84 [0.32-25.40], $p=0.350$ for MCP5 joints). There was no statistical significant difference between MMP-3 positive and MMP-3 negative patients for the increase in the number of bone erosions at the level of both feet or hand joints after the follow-up period (OR 1.35 [0.37-4.93], $p=0.647$ for wrist; OR 0.83 [0.22-3.09], $p=0.785$ for MCP2 joints; OR 3.30 [0.3729.1], $p=0.283$ for MCP5 and OR 2.44 [0.63-9.45], $p=$ 0.195 for MTP5 joints).

\section{US Assessment of Healthy Controls}

US finding of bone erosion was present in 4 (13.3\%) healthy persons. All were present at the level of lunate bone. The sensitivity of US bone erosions in RA diagnosis was $90.8 \%$ and specificity of $83.3 \%$ (AUC: 0.912 ).

\section{Radiographic Assessment}

After 2 years of treatment, only 6 (11.5\%) early RA patients developed bone erosions visible on X-ray. All of these erosions were confirmed by US examination. There was no statistically significant difference between MMP3 -negative and -positive patients for the modified TSS, at the baseline visit and after 24 months of follow-up ( $p=$ $0.43, p=0.63$ respectively; $p>0.05$ Mann-Whitney test).

\section{Disease Activity Assessment}

MMP-3-positive patients had significantly higher values of ESR, CRP, and DAS-28 than MMP-3-negative patients at the baseline evaluation $(p=0.002, p=0.005$, and 
$p=0.002$ respectively; $p<0.05)$. All of those parameters were significantly decreased after 24 months of treatment in the group of MMP-3-positive patients compared to the MMP-3-negative patients $(p=0.009, p=0.021$, and $p=$ 0.028 respectively; $p<0.05$; Table 4$)$.

\section{Discussion}

Diagnosing RA in the early stages of the disease and predicting its progression are essential in an everyday rheumatology practice $[18,19]$. It has been estimated that $60-90 \%$ of patients with RA have a progressive disease course [20]. Biomarkers of joint damage, such as a high level of MMP-3 could provide potentially important prognostic information about future structural destruction in patients with early RA $[9,21]$. High sensitivity and specificity of US examination in detecting bone erosions in RA diagnosis was found in our study. Moreover, bone erosions as detectable by X-ray were not present at study entry and only 6 (11.5\%) patients had evidence of erosion by X-ray after 24 months of follow-up, US evaluation of erosion was more sensitive; 55 patients $(87.3 \%)$ had bone erosions detected by US at study entry and all of patients after 24 months of follow-up.

To the best of our knowledge, this is one of the first prospective studies to investigate the association of serum levels of MMP-3 and development of bone erosions detected by US. In our study, US assessments showed that MMP-3-positive patients with early RA had a higher total number of bone erosions compared to MMP-3-negative patients at the baseline visit but without a statistically significant difference. After 24 months of treatment, the total number of bone erosions in MMP-3-positive patients was significantly higher compared to the number of MMP-3-negative patients. None of the confounding factors tested contributed to this result. Furthermore, after the follow-up period, there was a statistically significant increase in the number of MMP-3-positive patients with bone erosions versus MMP-3-negative patients with no significant increase. Therefore, high baseline levels of MMP-3 could be an important risk factor for structural damage in early RA. However, further clinical studies are needed to support this conclusion.

Patients with higher serum baseline levels of MMP-3 than normal had more bone erosion after 24 months of treatment compared to MMP-3-negative, but the difference was not statistically significant. Using logistic regression analyses, MMP-3-positivity was not able to predict increase in number of bone erosions after 24 months. This can be explained by the fact that a high percentage

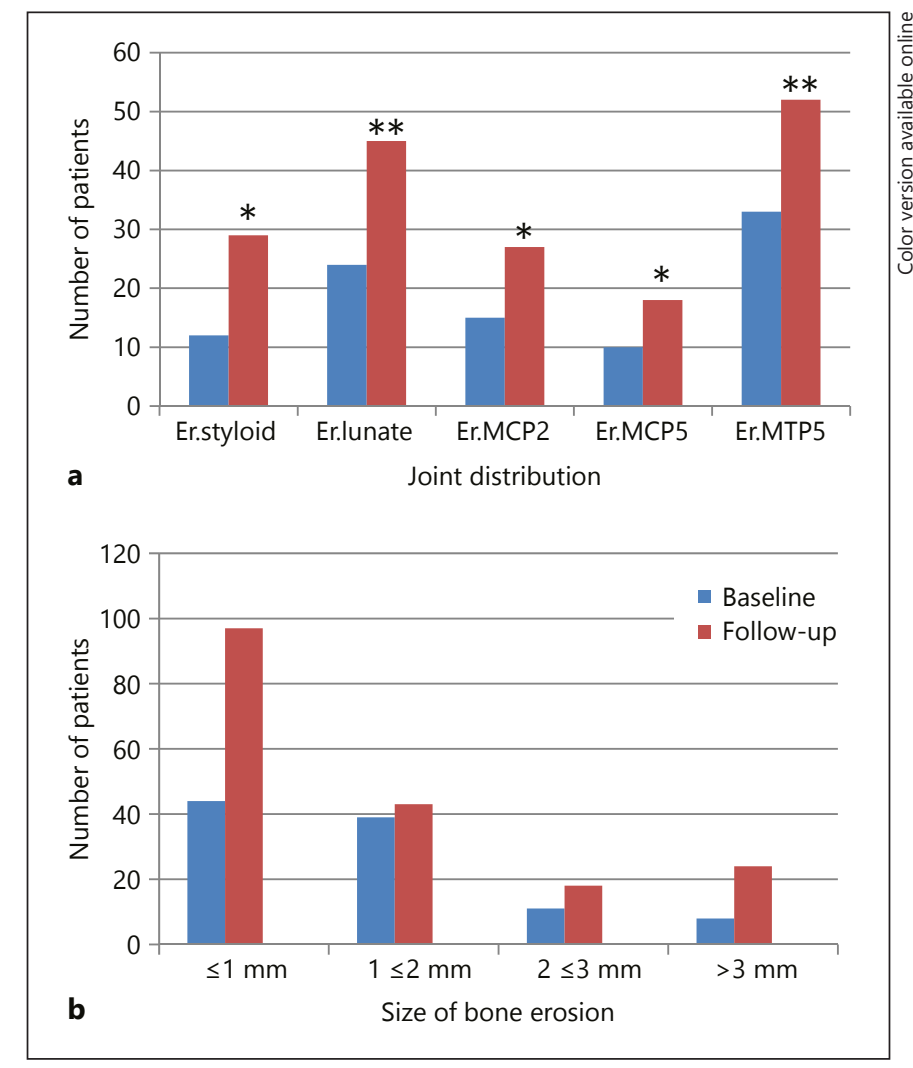

Fig. 1. a Number of bone erosions in analized joints (styloid process, lunate bone, MCP2, MCP5 and MTP5) in MMP-3-positive patients at baseline visit and after 24 months. b Size of bone erosion in analized joints (styloid process, lunate bone, MCP2, MCP5 and MTP5) in MMP-3-positive patients at baseline visit and after 24 months. a McNemar test. b Size of bone erosion $1=\leq 1 \mathrm{~mm}$; size of bone erosion $2=1 \leq 2 \mathrm{~mm}$; size of bone erosion $3=2 \leq 3$ $\mathrm{mm}$; size of bone erosion $4>3 \mathrm{~mm}$.

of patients (both MMP-3-positive and -negative) already had erosion as detected by US at baseline visit. Our study showed that baseline levels of MMP-3 had a predictive value in progression of size of bone erosions at the level of feet joints but not of hand joints. However, a longer follow-up period might show a similar result for the hand joints too. Furthermore, there was no significant difference between MMP-3-positive and MMP-3-negative patients for the increase in the number of bone erosions at the level of both feet and hand joints.

The majority of published clinical studies focused on the significance of the levels of MMP-3 for prediction of the radiographic progression in patients with RA [22-24]. In a 2-year follow-up study, Posthummus et al. [23] investigated the significance of levels of MMP-3 for structural joints damage in early RA and this study was the first to 
include patients without initial radiographic changes. Serum levels of MMP-3 in patients with early RA without damage as detected by X-ray at study entry were compared to levels of MMP-3 in patients with damage as detected by $\mathrm{X}$-ray. They found significantly higher baseline serum levels MMP-3 in patients with initial damage compared to the serum levels in the group without any damage at study entry and during follow-up. However, no difference in levels of MMP-3 was found between the group of patients who already had structural joint damage and the group of patients who developed damage during the follow-up. Gall et al. [25] followed DMARDs-naïve early RA patients for one year. At baseline they found a significant positive correlation between baseline levels of MMP-3 and the magnetic resonance imaging (US outcome measures in RA clinical trials) erosion scores, whereas no correlation was found with the Van Der Heijde modification of the Sharp scoring system (SvdH) and the baseline levels of MMP-3. After 12 months, the SvdH scores showed a significant correlation with the baseline levels of MMP-3. In a 12-month prospective study of early, DMARDs-naïve RA patients, Green et al. [21] found a strong correlation between baseline levels of MMP-3 and the progression of Larsen score. In a retrospective study, Mamehara et al. [26] found higher baseline levels of MMP-3 in a group of RA patients with radiographic progression without statistical significance. Our study focused on DMARDs-naïve early RA patients with $\leq 1$ year duration of symptoms, similar to the previous studies; however, the absence of structural damage detected by X-ray, required for our patient study enrollment, was the main difference from the previous ones. MMP-3-negative patients had higher modified TSS score then MMP3-positive patients at baseline visit, as well as after the follow-up, because of differences in joint space narrowing scores. A significant difference was not found in the modified TSS score between the MMP-3-positive and the MMP-3-negative patients after 2 years of treatment, which was different from the previous results. However, $15 \%$ of patients in Green's study, 39\% of patients in Posthummus study and $72 \%$ of patients in Gaal's study already had structural damage detected by X-ray at entry in contrast to our patients who were all without erosions visible on X-ray. In addition, our MMP-3-positive and -negative patients both had more active disease at study entry, which may also explain the finding of a high percentage of patients with bone erosion detected by US at the baseline visit. That was the reason why most of our patients were treated with methotrexate after baseline assessment and with biologics during the follow-up period unlike previous studies. This could further explain the lesser radiographic progression in all

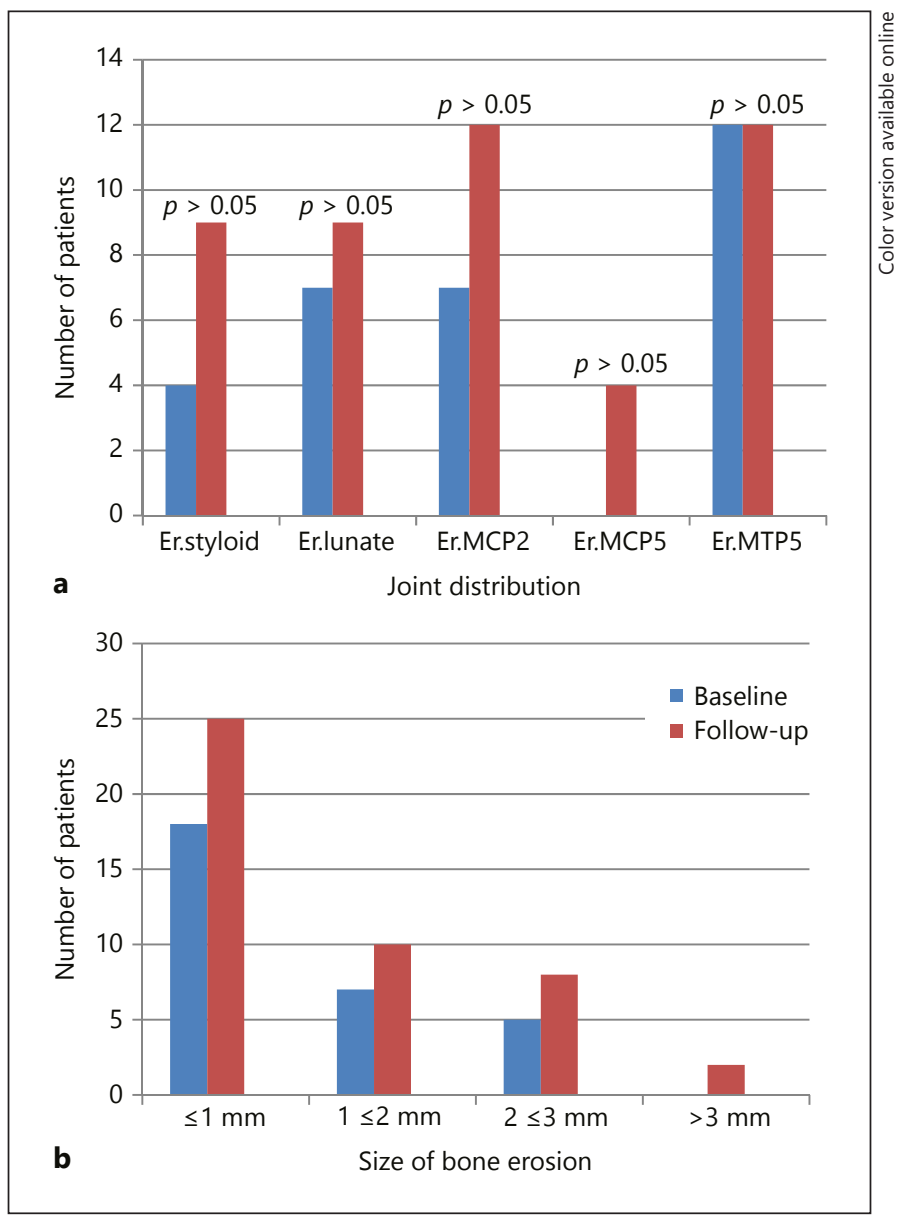

Fig. 2. a Number of bone erosion in analized joints (styloid process, lunate bone, MCP2, MCP5 and MTP5) in MMP-3-negative patients at baseline visit and after 24 months. b Size of bone erosion in analized joints (styloid process, lunate bone, MCP2, MCP5 and MTP5) in MMP-3-negative patients at baseline visit and after 24 months. McNemar test. Size of bone erosion $1=\leq 1 \mathrm{~mm}$; size of bone erosion $2=1 \leq 2 \mathrm{~mm}$; size of bone erosion $3=2 \leq 3 \mathrm{~mm}$; size of bone erosion $4>3 \mathrm{~mm}$.

patients in our group after 24 months of treatment especially in the group of MMP-3-positive patients who were treated more aggressively than the MMP-3-negative patients. Also, this explains the higher modified TSS score in the MMP-3-negative patients after 24 months. In contrast to previous studies, where the authors calculated the present serum level MMP1-3 in each patient, we divided patients into MMP-3-positive and -negative groups. In addition, matrix metalloproteinase- 3 gene polymorphism could also explain the differences mentioned above. It has been shown recently shown that the degree and rate of bone erosion damage are higher in RA patients with the $6 \mathrm{~A} / 6 \mathrm{~A}$ genotype [27]. 
Ally et al. [24] studied 128 DMARDs-naïve early RA patients and also So et al. [22] in one of the earliest studies, did not find a correlation between levels of MMP-3 and erosions detected by X-ray similar to our results. Serum levels of MMP-3 were significantly elevated in RA patients [28, 29 ] and strongly correlated with the levels of CRP and ESR [22-24, 27]. In our group, $73 \%$ of early RA patients showed increased levels of MMP-3, which were strongly correlated with the parameters of disease activity (ESR, CRP, DAS-28) and patients' disability (HAQ). The decrease of these parameters was significantly higher in the group of MMP3 -positive patients after 24 months of treatment. These results are similar to previously published results and emphasize the usefulness of MMP-3 in assessing disease activity in the early stages of RA as a marker of joint inflammation as well as the role of this biomarker in monitoring treatment efficiency [3, 21, 24, 29-31].

\section{Conclusion}

After 2-year of follow-up, ultrasound assessment showed a higher number of new bone erosions in MMP3-positive compared to MMP-3-negative patients with early RA and no visible initial radiographic changes. High baseline serum levels MMP-3 predict significantly higher progression of structural damage at the level of feet joints but not at the level of hand joints.

\section{References}

1 Al-Herz A, Al-Awadhi A, Saleh K, et al: Low prevalence of nodules in rheumatoid arthritis patients in Kuwait: a description and a comparison of patients from the Kuwait Registry for Rheumatic Diseases. Med Princ Pract 2017;26:152-156.

2 Fuchs HA, Kaye JJ, Callahan LF, Nance EP, PIncus T: Evidence of significant radiographic damage in rheumatoid arthritis within the first 2 years of disease. J Rheumatol 1989;16: 585-591.

3 Tchetverikov I, Lard LR, Degroot J, et al: Matrix metalloproteinases-3, $-8,-9$ as markers of disease activity and joint damage progression in early rheumatoid arthritis. Ann Rheum Dis 2003;62:1094-1099.

4 Soubrier M, Dougados M: How to assess early rheumatoid arthritis in daily clinical practice. Best Pract Res Clin Rheumatol 2005;19:73-89.

5 Brown AK, Conaghan PG, Karim Z, et al: An explanation for the apparent dissociation between clinical remission and continued structural deterioration in rheumatoid arthritis. Arthritis Rheum 2008;58:2958-2967.

6 Houseman M, Potter C, Marshall N, et al: Baseline serum MMP-3 levels in patients with Rheumatoid Arthritis are still independently predictive of radiographic progression in a longitudinal observational cohort at 8 years follow-up. Arth Res Ther 2012;14:R30.

7 Ribbens C, Porras MM, Franchimont N, et al: Increased matrix metalloproteinase- 3 serum levels in rheumatic diseases: relationship with synovitis and steroid treatment. Ann RheumDis 2002;61:161-166.

8 Kobayashi A, Naito S, Enomoto H, et al: Serum levels of matrix metalloproteinase 3 (stromelysin 1) for monitoring synovitis in rheumatoid arthritis. Arch Pathol Lab Med 2007;131:563-570.

9 Dorr S, Lechtenbohmer N, Rau R, et al: Association of a specific haplotype across the genes MMP1 and MMP3 with radiographic joint destruction in rheumatoid arthritis. Arthritis Res Ther 2004;6:R199-R207.

10 Narváez JA, Narváez J, De Lama E, De Albert M: MR imaging of early rheumatoid arthritis. Radio Graphics 2012;30:143-165.

11 Damjanov N, Radunovic G, Prodanovic S, et al: Construct validity and reliability of ultrasound disease activity score in assessing joint inflammation in RA: comparison with DAS28. Rheumatol (Oxford) 2012;51:120-128.

12 Døhn UM, Terslev L, Szkudlarek M, et al: Detection, scoring and volume assessment of bone erosions by ultrasonography in rheumatoid arthritis: comparison with CT. Ann Rheum Dis 2013;72:530-534.

13 Wakefield RJ, Balint PV, Szkudlarek M, et al: Musculoskeletal ultrasound including definitions for ultrasonographic pathology. J Rheumatol 2005;32:2485-2487.

14 Rahmani M, Chegini H, Najafizadeh SR, et al: Detection of bone erosion in early rheumatoid arthritis: ultrasonography and conventional radiography versus non-contrast magnetic resonance imaging. Clin Rheumatol 2012;29:883-891.

15 Aletaha D, Neogi T, Silman AJ, et al: 2010 Rheumatoid arthritis classification criteria: an American college of rheumatology/European league against rheumatism collaborative initiative. Ann Rheum Dis 2010;69:1580-1588.

16 Wakefield RJ, Balint PV, Sckudlarek M, et al: OMERACT 7 Special Interest Group Musculoskeletal ultrasound including definitions for ultrasonographic pathology. Rheumatol 2005;32:2485-2487.

17 Van Der Heijde DM: Plain X-rays in rheumatoid arthritis: overview of scoring methods, their reliability and applicability. Baillieres Clin Rheumatol 1996;10:435-453.

18 Van Nies JA, de Jong Z, van der Helm-van Mil $\mathrm{AH}$, et al: Improved treatment strategies reduce increased mortality risk early RA patients. Rheumatol (Oxford) 2012;49:2210-2216.
19 van der Heijde D, Klareskog L, RodriguezValverde V, et al: Comparison of etanercept and methotrexate, alone and combined, in the treatment of rheumatoid arthritis: twoyear clinical and radiographic results from the TEMPO study, a double-blind, randomized trial. Arthritis Rheum 2006;54:10631074.

20 Emery P, Mcinnes IB, VanVollenhoven R, Kraan MC: Clinical identification and treatment of a rapidly progressing disease state in patients with rheumatoid arthritis. Rheumatology 2008;47:392-398.

21 Green MJ, Gough AK, Devlin J, et al: Serum MMP-3 and MMP-1 and progression of joint damage in early rheumatoid arthritis. Rheumatol (Oxford) 2003;42:83-88.

22 So A, Chamot AM, Peclat V, et al: Serum MMP-3 in rheumatoid arthritis: correlation with systemic inflammation but not with erosive status. Rheumatol 1999;38:407-410.

23 Posthummus MD, Limburg PC, Westra J, et al: Serum levels of matrix metallproteinase- 3 in relation to the development of radiological damage in patients with early rheumatoid arthritis. Rheumatology 1999;38:1081-1087.

24 Ally MM, Hodkinson B, Meyer PW, et al: Serum matrix metalloproteinase- 3 in comparison with acute phase proteins as a marker of disease activity and radiographic damage in early rheumatoid arthritis. Mediators Inflamm 2013;2013:183653.

25 Gall SMA, El-Shafey AM, Hagrass HA, et al: Baseline serum level of matrix metalloproteinase- 3 as a biomarker of progressive joint damage in rheumatoid arthritis patients. Int J Rheum Dis 2014;14:261-266.

26 Mamehara A, Sugimoto T, Sugiyama D, et al: Serum matrix metalloproteinase- 3 as predictor of joint destruction in rheumatoid arthritis, treated with non-biological disease modifying anti-rheumatic drugs. Kobe J Med Sci 2012;56:E98-E107.
Serum MMP-3 Levels in RA Patients without Initial Radiographic Changes 
27 Ma MJ, Liu HC, Qu XQ, Wang JL: Matrix metalloproteinase-3 gene polymorphism and its mRNA expression in rheumatoid arthritis. Genet Molec Res 2015;14:15652-15659.

28 Posthumus MD, Limburg PC, Westra J, van Leeuwen MA, et al: Serum matrix metalloproteinase 3 levels in comparison to C-reactive protein in periods with and without progression of radiological damage in patients with early rheumatoid arthritis. Clin Exp Rheumatol 2003;21:465-472.

29 Sun S, Bay-Jensen AC, Karsdal MA, et al: The active form of MMP-3 is a marker of synovial inflammation and cartilage turnover in inflammatory joint diseases. BMC Musculoskelet Disord 2014;15:93.

30 Posthumus MD, Limburg PC, Westra J, et al: Serum matrix metalloproteinase 3 in early rheumatoid arthritis is correlated with disease activity and radiological progression. J Rheumatol 2000;27:2761.

31 Zhou L, Wang G, Liu X, et al: Matrix metalloproteinase-3 and the 7-joint ultrasound score in the assessment of disease activity and therapeutic efficacy in patients with moderate to severe rheumatoid arthritis. Arthritis Res Ther 2017;19:250. 